\title{
MobiAct: Supporting Personalized Interaction with Mobile Context-aware Applications
}

\author{
Adrian Stoica and Nikolaos Avouris
}

\author{
University of Patras, Department of Electrical and Computer Engineering, \\ Human Computer Interaction Group, \\ Eratosthenous Street, 26500 Rio-Patras, Greece \\ \{stoica,avouris\}@upatras.gr
}

\begin{abstract}
In this paper we present a conceptual framework for interaction with mobile context aware applications. The framework focuses especially on public and semi-public environments. Based on this framework a generic abstract architecture has been designed and several of its parts have been implemented. We discuss the implications and the support that this architecture provides for personalization of interaction. The architecture supports high interoperability and flexibility, with capability of tackling issues like privacy and degree of user control. The framework has been tested in typical spaces: a library and a museum. The paper concludes with a set of examples of use of the defined framework that cover typical situations for intra-space and across spaces usage.
\end{abstract}

\section{Introduction}

People need to access information and resources where and when they are performing their activities to achieve the multitude of daily tasks in a satisfactory manner. Today mobile devices are used widely to provide access to information and services associated with tangible objects through various technologies (RFID tags, two dimensional optical codes, Bluetooth etc.). As a result, an increasing number of context aware applications exist in public environments which provide various services and information to the public when and where they are needed.

Many institutions that have responsibility for places of public interest (libraries [1], museums [2], showrooms [5], schools [4], supermarkets [6] etc.) introduce in their environments extensions for mobile applications to harness the new potential technology brings. The physical space owned by these institutions is gradually enriched by and interweaved with a digital information space. Users (visitors, clients, readers etc) need to interact with both spaces to fully benefit of all offered

Please use the following format when citing this chapter:

Stoica, A. and Avouris, N., 2009, in IFIP International Federation for Information Processing, Volume 296; Artificial Intelligence Applications and Innovations III; Eds. Iliadis, L., Vlahavas, I., Bramer, M.; (Boston: Springer), pp. 389-397. 
services to them and inherently they need devices that can link the physical and digital spaces.

Personalization and adaptation of these services to the users are very relevant in this context, however a number of issues mostly related to security and privacy [7] need to be addressed.

Mobile applications can exploit user profiles as valuable information that can dramatically improve their quality and their relevance. User profiles are based on user traces, logs, user selections (small surveys completed, search terms etc), frequency of use of various features. While the information can be used to improve the quality and the efficiency of the services, there is a risk to be used against the user. People are concerned about privacy. They wish to control their own data and they do not like to feel followed. Usually users want the benefits of an adaptive system that gives them relevant feedback but, on the other hand, they do not like that the system gathers data about them.

The system design must take into account that systems and applications must support and facilitate the tasks and the activities of their users. Users must feel that they are in control of the system and they should fully understand the benefits and the trade-offs of using it.

The user profile can be common to more applications using an external user modelling server to allow interoperability [3]. This user profile, which is composed of the traces that the user leaves in the visited places, is enriched and updated according to the security and privacy options selected.

Middleware for context aware applications have been developed to allow fast and consistent deployment of such applications. The existing middleware approaches are tackling issues like context management $[8,14,15]$, privacy and security [11], collaboration and social interaction [10], data sharing [9], service discovery and so on.

For context aware mobile applications to step out from the labs and to be widely used by the general public, it is required to find solutions for consistent management of the service providers and consumers, user profiles and support of heterogeneity of mobile devices' platforms.

\section{The MOBIle InterACTion Framework - MobiAct}

The key concepts of our MobiAct framework are: hybrid space, physical hyperlink, hybrid space interaction device, the context and the dynamic service binding.

Hybrid Space is the space obtained from the fusion of physical and virtual spaces that are interweaved. The need to access the virtual space comes from the actual tasks the users have to perform in a certain physical space. While physical space knowledge can be accessed using perceptual mechanisms, the virtual space needs a mobile device to fetch and present information in a form suitable for human processing. 
Almost any physical artefact has associated information/services from different providers. The relevance of the information depends in some extent on the relation that the provider has with the physical space where the artefact is found - e.g. ownership.

The physical world artefacts and the virtual space items are linked together by an unequal correspondence (e.g. a very small physical object can have linked to it many digital objects).

To instantiate the link between the physical and virtual artefacts, we need means of interacting with the real world $[12,13]$. From the need of this kind of hybrid interactions the concept of physical hyperlink or object hyperlinking emerged.

A Physical Hyperlink, as a means to connect a physical artefact with the digital information/ services associated to it that uniquely identifies the physical artefact or a class of equivalent artefacts. A physical hyperlink can be implemented using several modes:

- Human readable visual cue (numbers, letters)

- Machine readable visual cue (2D, 1D barcodes)

- RF tag (RFID, Bluetooth)

The different implementations of physical hyperlinks have various aspects that match characteristics of different mobile devices. Considering this issue, we propose that a standardized multi-modal approach to be adopted for implementing physical hyperlinks. Including a human readable visual cue will ensure mass accessibility provided that many devices support text entry. In figure 1 the book physical hyperlink includes modalities for barcode scanners, camera equipped devices and human visual perception.

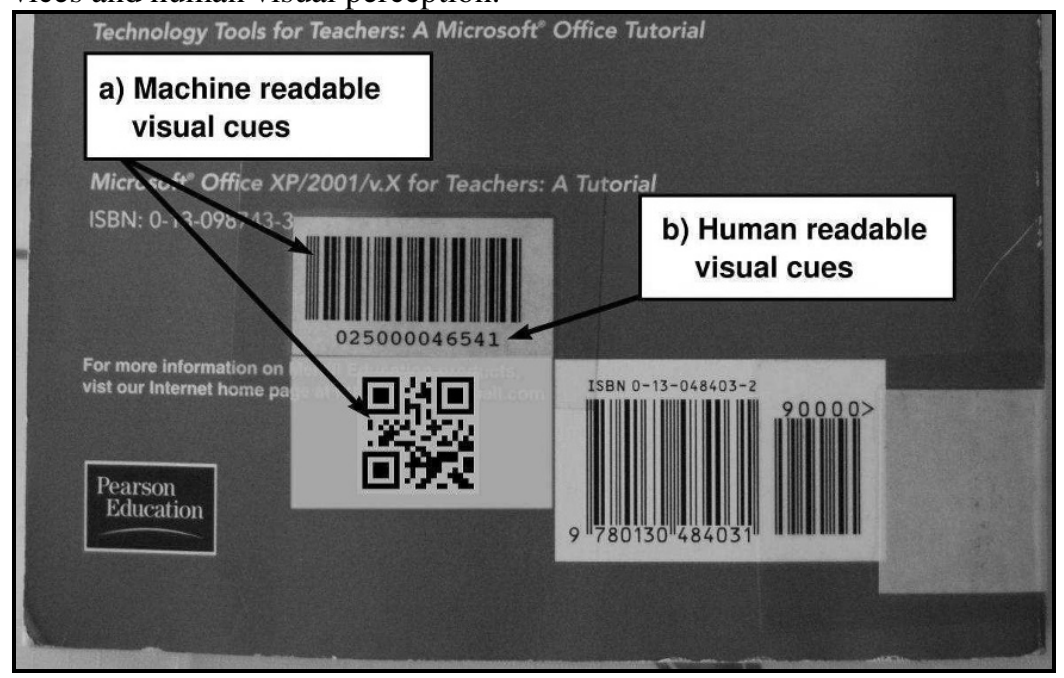

Fig. 1. Multimodal physical hyperlink attached to a library book 
To access the virtual part of the hybrid space a device is needed. We call this device Hybrid Space Interaction Device (typical such devices are a mobile phone or a PDA) and we identified the following requirements:

- Main requirements: Some sort of network connectivity (GPRS, 3G, WiFi), Ability to run third party applications, Basic Input/Output abilities (screen, keyboard, touchscreen, stylus);

- Optional requirements: Camera, RFID reader, Barcode scanner, Other kind of sensors.

Context is very important in mobile interaction because the applications can use it to adapt and filter the relevant information. Context elements are for example: device type and capabilities, user profile, actual task, state of the space, modality of interaction, location.

Another important concept in our proposal is dynamic service binding. The dynamic service binding is connecting the physical hyperlinks with the appropriate services and/or information. The choice of services delivered to the user upon selecting a certain physical hyperlink is produced by fusing several elements from the context of use - identity of the user, user preferences or profile, application provider, device used, actual task.

An example of context of use involvement in physical hyperlink selection outcome is the following: in a museum the selection of a physical hyperlink can trigger displaying of textual information or playing audio narrations if in guide mode or could trigger collection of hints, creation of links etc. if in educational game mode.

Services provided for the hybrid space user fall in the following categories: informational, transactional, navigational, control.

\section{MobiAct Architecture}

Based on the MobiAct framework we have designed an architecture that takes into account among others: roaming among contexts and spaces, personalization, anonymity, privacy and security.

People move every day through a succession of public, semi-public and private spaces according to their goals and their activities. The identity of the people sometimes should be known to grant them access to certain spaces, while some other times, it should be hidden to protect user privacy.

The relevance of accessing mobile services highly depends on user goals and the nature of the tasks she is doing. The utility a mobile service provides to a user depends on users' interests, goals and lifestyle.

The figure 2 depicts the proposed MobiAct architecture. The user is in a hybrid enabled space and she performs a certain task. In the physical space physical artefacts are present which allow user interactions through physical hyperlinks. 
The main entities in our architecture are: user, user agency, user agent, physical space administrator/owner, semantics of space service, broker.

The figure 2 focuses on a certain physical space area at a certain moment. The left side presents the physical space, while the right side presents the virtual space elements that are separated by a dashed line for the sake of clarity. The entities "User Agency", "Broker" are represented as single instance for the simplicity of the schema. A different user could use another agency or maybe she could use several agencies. In the same way, there can be more brokers competing between them in the quality of service providing. However, at a certain moment of interaction, the user can be associated with one "User Agency" and she deals with one "Broker".

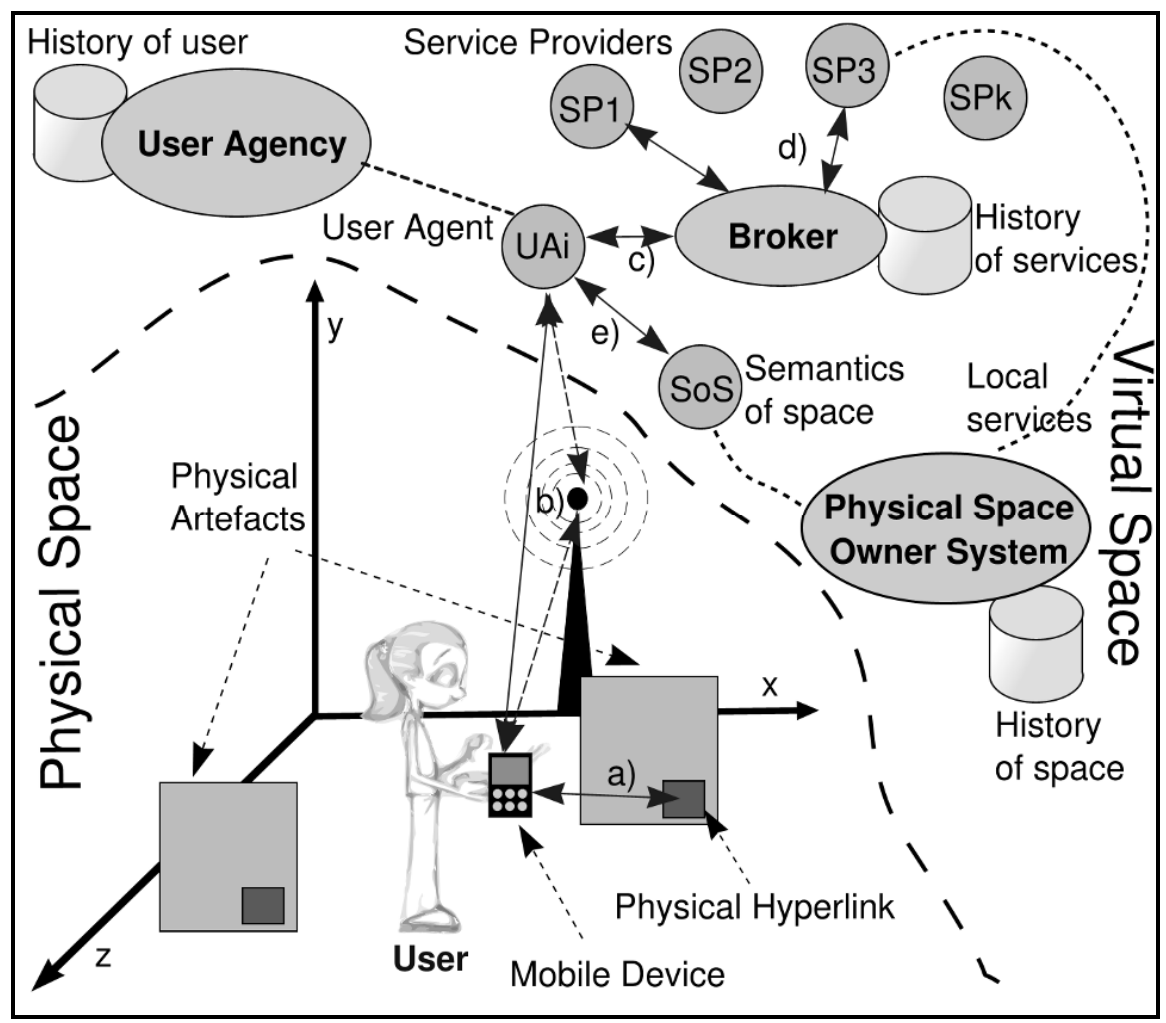

Fig. 2. MobiAct Architecture

The user is represented in the virtual space by "User Agent" that mediates interaction with other entities in the virtual space. The user initiates interaction with an artefact and a typical sequence of actions takes place. Let us examine the scenario of interacting with the hybrid space.

There are several phases of interaction. First there is the initiation phase where the user starts the interaction with an artefact. The user agent has to select a Bro- 
ker suitable for the space, making use of previous interaction trails from the user to propose a set of available service providers to the user.

After selecting the desired service provider the interaction advances in the second phase of service consuming. During her interaction with one service provider the user might change its goals or she might understand that this service is not what she needed. As a result she might change back to the initiation phase.

We have identified the following typical sequence of events during initial phase of interaction with a hybrid space:

- The user is utilizing her mobile device to interact with the physical hyperlink of an object (physical artefact);

- The device software connects with the user agent - there are two possibilities: 1) the user connects by means of an independent network like a $3 \mathrm{G}$ provider or

2) the user connects using a network provided by the space administrator/owner - to request services for the selected object.

- The user agent selects a relevant broker (by means of a sort of directory service) and it issues a request for service providers based on the user profile and actual task performed and possibly on semantics of space provider information.

- Further on, the broker issues a request to available service providers registered for that space. Upon the offers from the service providers it supplies the user agent with a set of service providers.

- The user agent utilizes user profile and information from the semantics of space provider to filter the results and to send them to the user.

\section{Personalization and Adaptation in MobiAct}

In MobiAct there are two levels for providing personalization and adaptation: intra-space and across spaces. The key element is the "User Agency" that is a trusted entity which the user herself selects. The "User Agency" provides the user with a ubiquitous accessible profile and also allows anonymous use of certain services.

There are three types of profiles that play a role in the MobiAct architecture: user profiles, hybrid space profiles and service provider profiles. During user interaction with a hybrid space there are generated trails of interaction that enrich these profiles. Except interaction trails profiles contain identity, interest ontology, generic user information (e.g. language(s), age, sex etc.) and privacy preferences for the user, rankings for hybrid space, content and service providers

At intra-space level the user experience is improved within a certain hybrid space through filtering and ranking content and service providers according to the user profile, community produced ratings, providers' and content's metadata and collective profile. Both user profile and provider's metadata are being built incrementally. 
The relations between the service provider and the physical space the user is immersed in influence the degree of relevance - e.g. In a museum the information provided by the museum service provider (information about exhibits) should be of higher precedence to other service providers

At across spaces level the architecture uses collective profiles and statistical methods to filter and rank content and service providers in new spaces based on trails from other spaces richer in trails and with a more complete profile.

The user agency has access to a multitude of user profiles in a multitude of spaces.

The adaptation of the services for the users is done through the combination of the personal model and the collective model with different weights according to the richness of the personal user model in the specific space.

In figure 3 a structured representation of the information in the user profile database is shown. When a user visits a new space the system can examine the specific context to find popular artefacts, information or activities. Also to match interest across spaces it can examine the interests already depicted in other contexts and to match them against the other users that have also interests defined for our current user new context.

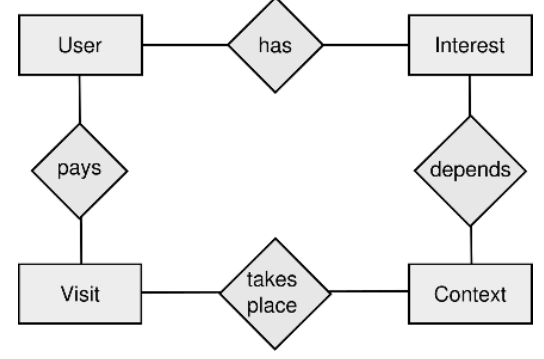

Fig. 3. Simplified ERD of user profile

The sum of trails of interaction over time builds up the history of the user, of the space and of services. Each interaction generates trails on the side of every participating entity. Using historical data each entity can improve its performance.

The visits of the various users in a certain space build up the specific space model. This model has a twofold use: on one hand provides a base for a collective statistical user model for new users and on the other hand provides information for restructuring both the physical and virtual space.

\section{Conclusion}

The MobiAct framework and the corresponding architecture aim at offering an application independent solution for mobile service providing and consuming. Introducing the User Agency entity the architecture allows not only intra-space but also across spaces personalization and adaptation. It aims at providing readily implemented functionality for the common features of context aware mobile application infrastructure. The benefits of this approach are related to cost and development effort for deployment of mobile services, as well as interoperability and fluidity of user experience across multiple spaces. In this paper the main characteristics of the MobiAct framework and architecture have been outlined together with its uses for personalization and adaptation. 
The roadmap for defining this architecture and developing the first prototype involved first gathering of requirement from two quite different semi-public spaces, a large Academic Library and a Cultural Historical Museum. During the requirements gathering phase, which involved stakeholder analysis and focus groups that discussed issues of privacy and service personalization, the two applications have been defined. After we will finalize the evaluation of the framework for intra-space use will follow the across spaces evaluation phase. When the final prototypes are developed, the privacy and trust issues related to the personalization functionality of the architecture will be tested with real users who are going to visit the two sites and experience the effect of different privacy policies on the quality of the service.

Acknowledgments Special thanks are due to the Hybrid Libraries Project, funded under the PENED 2003 Program, Grant number 03ED791 by the General Secretariat of Research and Technology for financial support and to the Project of Supporting visitors of Solomos Museum, funded by the Museum of Solomos and Prominent Zakynthians, under the Information Society Program, for providing us with application requirements for personalization and adaptation of the developed services.

\section{References}

1. Aittola M, Ryhänen T \& Ojala T (2003) SmartLibrary - Location-aware mobile library service. Proc. 5th International Symposium on Human Computer Interaction with Mobile Devices and Services, Udine, Italy, 411-416.

2. Exploratorium (2005), Electronic Guidebook forum Report, San Francisco, Available at: www.exploratorium.edu/guidebook/eguides_forum2005.pdf Accessed: April 2006

3. Heckmann D., Schwartz T., Brandherm B., Schmitz M., and von WilamowitzMoellendorff M., GUMO - The General User Model Ontology, L. Ardissono, P. Brna, and A. Mitrovic (Eds.): UM 2005, LNAI 3538, pp. 428-432, 2005.

4. Liang J.-K., Liu T.-C., Wang H.-Y., Chang B., Deng Y.-C., Yang J.-C., Chouz C.-Y., Ko H.-W., Yang S., \& Chan T.-W., A few design perspectives on one-on-one digital classroom environment, Journal of Computer Assisted Learning 21, pp181-189

5. mEXRESS Project Homepage, available at http://mexpress.intranet.gr/index.htm, last accessed October 2006

6. Roussos G., Koukara L., Kourouthanasis P, Tuominen J.O., Seppala O., Giaglis G. and Frissaer J., 2002, "A case study in pervasive retail", ACM MOBICOM WMC02, pp. 90-94.

7. SWAMI Project (2006), Dark scenarios in ambient intelligence: Highlighting risks and vulnerabilities, Deliverable D2, Available: http://swami.jrc.es/pages/documents/ SWAMI_D2_scenarios_Final_ESvf_003.pdf Accessed: April 2006

8. Dey, A.K., Salber, D., Abowd, G.: A Conceptual Framework and a Toolkit for Supporting the Rapid Prototyping of Context-Aware Applications. Human-Computer Interaction 16 (2001) 97-166

9. Boulkenafed M, Issarny V, A Middleware Service for Mobile Ad Hoc Data Sharing, Enhancing Data Availability, Middleware 2003 (2003), pp. 493-511.

10. Kern S, Braun P, Rossak W, MobiSoft: An Agent-Based Middleware for Social-Mobile Applications, On the Move to Meaningful Internet Systems 2006: OTM 2006 Workshops (2006), pp. 984-993. 
11. Heckmann D, Ubiquitous User Modeling, Vol. 297, Dissertations in Artificial Intelligence, IOS Press, Amsterdam, NL, 2006

12. E. Rukzio, M. Paolucci, T. Finin, P. Wisner, T. Payne (Eds.), Proceedings Workshop Mobile Interaction with the Real World (MIRW 2006), available at http://www.hcilab.org/events/mirw2006/pdf/mirw2006_proceedings.pdf, retrieved on 11th of April 2008

13. G. Broll, A. De Luca, E. Rukzio, C. Noda, P. Wisner (Eds.), Proceedings Workshop Mobile Interaction with the Real World (MIRW 2007), available at http://www.medien.ifi.lmu.de/pubdb/publications/pub/broll2007mirwmguidesTR/broll200 7mirwmguidesTR.pdf, retrieved on 11th of April 2008

14. Riva O, Contory: A Middleware for the Provisioning of Context Information on Smart Phones, Middleware 2006 (2006), pp. 219-239.

15. Zimmermann A., Specht M. and Lorenz A., Personalization and Context Management, User Modeling and User-Adapted Interaction Special Issue on User Modeling in Ubiquitous Computing, Vol. 15, No. 3-4. (August 2005), pp. 275-302. 